\title{
A characterization of point shadows of residues in building geometries
}

\author{
Anna Kasikova
}

full screen

close

\section{Abstract}

We characterize shadows of residues in building shadow spaces by local properties. The results are applied to buildings with diagram $\mathrm{Y}_{l, m, n}$ (including $\mathrm{E}_{k}, k \in\{6,7,8\}$, and $\mathrm{D}_{k}$ ) to show that every subspace isomorphic to the shadow of a residue is the shadow of a residue.

Keywords: building, incidence geometry, point-line geometry, shadow space, building Grassmannian

MSC 2010: 51E24

\section{Introduction and definitions}

There are many characterizations of shadows of residues and apartments of residues in building shadow spaces seemingly dependent on the specific geometry (e.g. $[3,2,7]$ ). In the present paper we give a general characterization of shadows of residues in a building shadow space (Theorems 2.1 and 3.1) and then apply our results to buildings with diagram $Y_{l, m, n}$ (Theorem 6.1). As an immediate corollary we obtain the following (for notation see Subsection 1.2).

Corollary 1.1. Suppose $\mathcal{B}$ is a building with Coxeter diagram $M$ over a set $I$ and $\Sigma=(\mathcal{P}, \mathcal{L})$ is its shadow space. Let $\phi: \Sigma\left|\mathcal{P}_{O} \rightarrow \Sigma\right| \mathcal{P}_{1}$ be an isomorphism, where $O$ is a residue of $\mathcal{B}$ and $\mathcal{P}_{1}$ is a subspace of $\Sigma$. Below, all subscripts are positive integers.

(1) Suppose $\Sigma$ is of type $\mathrm{A}_{n, k}$ with $n \geq 3$ and $1 \leq k \leq n$, of type $\mathrm{D}_{n, k}$ with $n \geq 4$ and $k \in\{1, n, n-1\}$, or of type $\mathrm{E}_{n, k}$ with $n \in\{6,7,8\}$ and $k \in\{1,2, n\}$. Then $\mathcal{P}_{1}=\mathcal{P}_{O^{\prime}}$ for a residue $O^{\prime}$ of $\mathcal{B}$ with $M_{O} \cong M_{O^{\prime}}$, where the isomorphism stabilizes the point node. 





\section{Characterization of shadows of residues: Theo- rem 2.1}

Theorem 2.1. Suppose hypothesis (A) holds and let $T \in \mathcal{T}$ be such that $|T| \geq 2$. Suppose $\Sigma_{1}=\left(\mathcal{P}_{1}, \mathcal{L}_{1}\right)$ is a connected full subgeometry of $\Sigma$ satisfying conditions (Shd-loc) and (Shd-pln):

(Shd-loc) For every $p \in \mathcal{P}_{1}$ there is an object $O_{p}$ of $\Gamma$ on $p$ with $\operatorname{typ}_{\#}\left(O_{p}\right)=T$ such that $\mathcal{L}_{p} \cap \mathcal{L}_{1}=\mathcal{L}_{p} \cap \mathcal{L}_{O_{p}}$.

(Shd-pln) For every pair of distinct collinear points $p$ and $q$ of $\Sigma_{1}$, and for every plane $\pi \in \Pi$ incident with $p$ and $q$, we have $\mathcal{L}_{1} \cap \mathcal{L}_{p} \cap \mathcal{L}_{\pi}-\{\langle p, q\rangle\} \neq \emptyset$ if and only if $\mathcal{L}_{1} \cap \mathcal{L}_{q} \cap \mathcal{L}_{\pi}-\{\langle p, q\rangle\} \neq \emptyset$.

Then $\Sigma_{1}=\Sigma \mid \mathcal{P}_{O}$, where $O \in \mathcal{O}$ and $\operatorname{typ}_{\#}(O)=T$.

We need two lemmas.

Lemma 2.2. Suppose the hypothesis of Theorem 2.1 holds and let $p, q \in \mathcal{P}_{1}$ be distinct collinear points of $\Sigma_{1}$.

(i) If $|T|=2$, then $O_{p}=O_{q}$.

(ii) If $|T| \geq 3$, then $\Pi_{\langle p, q\rangle} \cap \Pi_{O_{p}}=\Pi_{\langle p, q\rangle} \cap \Pi_{O_{q}}$.

Proof. By condition (Shd-loc) $O_{p}$ and $O_{q}$ are both incident with $\langle p, q\rangle$. If $|T|=2$, let $\pi=O_{p}$; if $|T| \geq 3$, let $\pi \in \Pi_{\langle p, q\rangle} \cap \Pi_{O_{p}}$.

By [4, Lemma 5.6(2)] applied to $\pi$, there is a line $L$ of $\pi$ on $p$ distinct from $\langle p, q\rangle$. By Remark 1.3(1) $L \in \mathcal{L}_{O_{p}}$, therefore by condition (Shd-loc) $L \in \mathcal{L}_{1}$. By condition (Shd-pln) there is a line $N \in \mathcal{L}_{1} \cap L_{q} \cap \mathcal{L}_{\pi}-\{\langle p, q\rangle\}$, and by condition (Shd-loc) $N \in \mathcal{L}_{O_{q}}$. Since $\langle p, q\rangle$ and $N$ are distinct lines of $\pi$ on $q$ incident with $O_{q}$, by [4, Lemma 5.6(1)] $\pi \subseteq O_{q}$.

If $|T|=2$ we have shown that $O_{p}=O_{q}$. If $|T| \geq 3$, then the above shows that $\Pi_{\langle p, q\rangle} \cap \Pi_{O_{p}} \subseteq \Pi_{\langle p, q\rangle} \cap \Pi_{O_{q}}$; the reverse inclusion follows by symmetry.

Lemma 2.3. Assume that the hypothesis of Theorem 2.1 holds and $|T| \geq 2$. Then, for all $p, q \in \mathcal{P}_{1}$, we have $O_{p}=O_{q}$.

Proof. Since $\Sigma_{1}$ is connected it suffices to show that $O_{p}=O_{q}$ for any two distinct collinear points $p$ and $q$ of $\Sigma_{1}$. For $|T|=2$ this is Lemma 2.2(i). Suppose $|T| \geq 3$. By condition (Shd-loc) $\langle p, q\rangle$ is incident with both $O_{p}$ and $O_{q}$. For $r \in\{p, q\}$, let $Z_{r}=r^{\#} \cap\langle p, q\rangle^{\#} \cap O_{r}^{\#}$; by Remark 1.3(5) and by property (Bld2) of buildings $Z_{r} \neq \emptyset$. Let $\mathcal{S}_{r}$ denote the set of all planes $\pi$ of $\Gamma$ such that $\pi^{\#} \cap Z_{r} \neq \emptyset$ and

$$
\operatorname{typ}_{\#}(\langle p, q\rangle) \subseteq \operatorname{typ}_{\#}(\pi) \subseteq T .
$$









with the minimality of $w$. The case $c^{\prime}=b^{\prime}$ is similar.

(7) The walks $u$ and $v^{-1}$ are geodesics from $p$ to $q$ in $\mathcal{G}$.

We prove the statement regarding $u$. By (1) $1(u)=1\left(u^{\prime \prime}\right)$. We show that $u^{\prime \prime}$ is a geodesic from $p$ to $q$ in $\mathcal{G}$. By (6) the gallery $U^{\prime \prime}$ lies in $R$. Let $\Delta=$ $\operatorname{ShSp}(R,\{i, j\})$; the points and lines of $\Delta$ are the chambers and panels of $R$ respectively, and the point-collinearity graph of $\Delta$ is the graph of the chamber system $R$ with the lables removed. Let $\psi: \Sigma \mid \mathcal{P}_{R} \rightarrow \Delta$ be the isomorphism of Remark 1.4; the image under $\psi$ of the walk $u^{\prime \prime}$ is $U^{\prime \prime}$. Since $U^{\prime \prime}$ is a geodesic in $R$, and by Remark 1.3(4) $\mathcal{P}_{R}$ is a convex subspace of $\Sigma$, $u^{\prime \prime}$ is a geodesic in $\mathcal{G}$.

(8) All vertices of $w$ are in $\mathcal{P}_{R}$.

By (7) $u$ and $v^{-1}$ are geodesics from $p$ to $q$ in $\mathcal{G}$, and by by Remark 1.3(4) $\mathcal{P}_{R}$ is a convex subspace of $\Sigma$, therefore (8) holds.

We can now finish the proof of the lemma. Let $\Delta$ and $\psi$ be as in the proof of (7) and let $G$ be the point-collinearity graph of $\Delta$; let $\mathcal{L}_{i}^{\prime}$ and $\mathcal{L}_{j}^{\prime}$ be the sets of the panels of $R$ of types $\{i\}$ and $\{j\}$ respectively. By (8) $\psi(w)$ is a shortest strictly $\left\{\mathcal{L}_{i}^{\prime}, \mathcal{L}_{j}^{\prime}\right\}$-circuit of $G$. Since $R$ is a generalized $m_{i j}$-gon, $\psi(w)=C$ for a circuit $C$ of $R$ of type $p(i, j) \circ p(j, i)^{-1}$. Then $w=\mathrm{w}_{\mathcal{G}}(C)$.

Corollary 4.6. Suppose hypothesis (A) holds, let $\{i, j\} \subseteq J$, and let $w$ be a strictly $\left\{\mathcal{L}_{i}, \mathcal{L}_{j}\right\}$-circuit of $\mathcal{G}$ of length $2 m_{i j}$. Then there exists a plane $\pi$ of $\Gamma$ with $\operatorname{typ}_{\#}(\pi)=\{i, j\}$ such that all vertices of $w$ lie in $\mathcal{P}_{\pi}$.

Proof. By Proposition 4.3 there is a circuit $C$ of $\mathcal{B}$ of type $p(i, j) \circ p(j, i)^{-1}$ such that $w=\mathrm{w}_{\mathcal{G}}(C)$. Let $\pi \in \Pi$ be such that $\operatorname{typ}_{\#}(\pi)=\{i, j\}$ and $\pi^{\#}$ contains $C$.

The following is immediate from Remark 1.4 and [5, Proposition 3.8(2)].

Lemma 4.7. Suppose hypothesis (A) holds, let $\{i, j\} \subseteq J$, and let $\pi$ be a plane of $\Gamma$ with $\operatorname{typ}_{\#}(\pi)=\{i, j\}$. Then $\Sigma \mid \pi$ is a generalized $2 m_{i j}$-gon with exactly two lines on each point; its point-collinearity graph is isomorphic to the graph of the residue $\pi_{\#}$ with the labels removed.

Proof of Proposition 3.3. By Lemma $4.7 \mathcal{G} \mid \mathcal{P}_{\pi}$ contains a strictly $\left\{\mathcal{L}_{i}, \mathcal{L}_{j}\right\}$-circuit $w_{0}$ of length $2 m_{i j}$. Let $w=\left(p_{0}, \ldots, p_{n}\right)$ be the image of $w_{0}$ under $\phi$. By (Phi-lin) $w$ is a strictly $\left\{\mathcal{L}_{i^{\prime}}, \mathcal{L}_{j^{\prime}}\right\}$-circuit of $\mathcal{G}$ of length $2 m_{i j}$, therefore by Corollary 4.6 there is a plane $\pi^{\prime}$ of $\Gamma$, such that $\operatorname{typ}_{\#}\left(\pi^{\prime}\right)=\left\{i^{\prime}, j^{\prime}\right\}$ and $\left\{p_{0}, \ldots, p_{n}\right\} \subseteq \mathcal{P}_{\pi^{\prime}}$. We claim that

(1) $\mathcal{P}_{1} \subseteq \mathcal{P}_{\pi^{\prime}}$ 



\section{Proof of Proposition 3.4}

Here and in Subsections 5.1-5.2 we prove preliminary results. We complete the proof of Proposition 3.4 in Subsection 5.3.

Lemma 5.1. Suppose the hypothesis of Proposition 3.4 holds. Then, for every object $U$ of $\Gamma_{O}^{-}$, $\phi$ induces an isomorphism $\Sigma\left|\mathcal{P}_{U} \rightarrow \Sigma\right| \mathcal{P}_{U^{\prime}}$, where $U^{\prime} \in \mathcal{O}$ and $\operatorname{typ}_{\#}\left(U^{\prime}\right)=\operatorname{typ}_{\#}(U)$.

full screen

close

quit

Proof. If $U$ is an object of the second kind, then $\left|\operatorname{typ}_{\#}(U)\right|<|T|$ and the restriction $\phi \mid \mathcal{P}_{U}$ satisfies the hypothesis of Theorem 3.1, therefore the conclusion holds by hypothesis. Suppose $U$ is an object of the first kind. Then by condition (Phi-str) of Theorem 3.1 there is $U^{\prime} \in \mathcal{O}$ such that $\phi\left(\mathcal{P}_{U}\right)=\mathcal{P}_{U^{\prime}}$ and $\operatorname{typ}_{\#}(U)=\operatorname{typ}_{\#}\left(U^{\prime}\right)$. By Remark 1.4, condition (Bld-iso), and condition (Bld-str), $U^{\prime}$ is as in the conclusion.

Corollary 5.2. Suppose the hypothesis of Proposition 3.4 holds. Then $\phi$ induces a monomorphism $\bar{\phi}: \Gamma_{O}^{-} \rightarrow \Gamma$ defined by, for all objects $U$ of $\Gamma_{O}^{-}, \bar{\phi}(U)=U^{\prime}$, where $U^{\prime}$ is as in Lemma 5.1 .

\subsection{An auxiliary proposition}

Suppose hypothesis (A) holds. Let $p \in \mathcal{P}$ and let $H \subseteq J$. We let $\Gamma(p, H)$ be the subgeometry induced in $\Gamma$ on the set of all objects $U$ of $\Gamma$ incident with $p$, that have the property that $\operatorname{typ}_{\#}(U) \cap J=H$. If $U$ is an object of $\Gamma(p, H)$, then its type in $\Gamma(p, H)$ is $\tau(U)-|H|$. The objects of $\Gamma(p, H)$ of types 1 and 2 will be called points and lines of $\Gamma(p, H)$ respectively. We denote $\Sigma(p, H)$ and $\mathcal{O}_{p, H}$ the point-line truncation and the set of objects of $\Gamma(p, H)$. For $U \in \mathcal{O}$ incident with $p$, we denote $\Gamma(p, H)_{U}^{-}$and $\Sigma(p, H)_{U}$ the subgeometries of $\Gamma(p, H)$ and $\Sigma(p, H)$ induced on the sets of objects belonging to $\Gamma_{U}^{-}$.

For $H \subseteq I$, define $\bar{H}:=\operatorname{typ}^{\#}(U)$, where $U \in \mathcal{O}$ and $\operatorname{typ}_{\#}(U) \subseteq H \subseteq$ $\operatorname{typ}^{\#}(U)$. Recall that $p^{\#}$ is an $I^{\prime}$-residue of $\mathcal{B}$. Suppose now that $H \subseteq J$. Let $H^{\prime}=I^{\prime} \cap \bar{H}, H^{\prime \prime}=I^{\prime}-H^{\prime}, \Phi(p, H)=\operatorname{ShGm}\left(p^{\#}, H^{\prime \prime}\right)$, and $\Delta(p, H)=$ $\operatorname{ShSp}\left(p^{\#}, H^{\prime \prime}\right)$. For $U \in \mathcal{O}$, we define $U_{p, H}=\operatorname{Sh}_{H^{\prime}}\left(U^{\#} \cap p^{\#}\right)$. For $T \subseteq I$ we let $T_{H}=\operatorname{typ}_{\#}\left(U_{p, H}\right)$ and $T^{H}=\operatorname{typ}^{\#}\left(U_{p, H}\right)$, where $U \in \mathcal{O}$ is such that $\operatorname{typ}_{\#}(U) \subseteq T \subseteq \operatorname{typ}^{\#}(U)$; both $T_{H}$ and $T^{H}$ are subsets of $I^{\prime}$.

The purpose of this subsection is to prove the following.

Proposition 5.3. Suppose the hypothesis of Proposition 3.4 holds. Let $p \in \mathcal{P}_{O}$, let $q=\phi(p)$, and let $H \subseteq J \cap T$. Suppose that (C1) or (C2) holds.

(C1) $|H|=1$ and $O_{p, H}$ is an object of $\Phi(p, H)$ of the first kind. 
(C2) $|H| \in\{1,2\}$ and $O_{p, H}$ is an object of $\Phi(p, H)$ of the second kind.

Then $\phi$ induces isomorphisms

(1) $\Sigma(p, H)_{O} \rightarrow \Sigma(q, H)_{O^{\prime}}$, where $O^{\prime} \in \mathcal{O}$ and typ $\#\left(O^{\prime}\right)=H \cup T^{H}$. (2) $\Delta(p, H)_{O_{p, H}} \rightarrow \Delta(q, H)_{O_{q, H}}$, where $O_{q, H}$ is an object of $\Phi(q, H)$ with $\operatorname{typ}^{\#}\left(O_{q, H}\right)=$
$T^{H}$.

go back

full screen

close

quit

The proof of Proposition 5.3 consists of Lemmas 5.4-5.9 below. First, we will show that Parts (1) and (2) of the conclusion are equivalent. Suppose hypothesis (A) holds. We say that $S \subseteq I$ is $I^{\prime}$-closed if $S=\operatorname{typ}^{\#}(U)$ for some $U \in \mathcal{O}$. For $S \subseteq I$ with $\operatorname{typ}_{\#}(U) \subseteq S \subseteq \operatorname{typ} \#(U)$ we let $\mathrm{Cl}_{I, I^{\prime}}(S):=\bar{S}$, where $\bar{S}$ is as defined before Proposition 5.3, and we call this the $I^{\prime}$-closure of $S$ in $I$. If $D$ is the diagram graph of $\mathcal{B}$ (that is, the diagram $M$ with the edge labels removed), then $\operatorname{typ}^{\#}(U)=\operatorname{typ}_{\#}(U) \cup\left(I^{\prime}-X\right)$, where $X=\cup\left\{D_{0,1}(k) \mid k \in\right.$ $\left.\operatorname{typ}_{\#}(U)\right\}$ and $D_{0,1}(k)$ denotes the set of vertices equal or adjacent to $k$ in $D$.

Lemma 5.4. Suppose hypothesis (A) holds, $D$ is the diagram graph of $\mathcal{B}$, and $H \subseteq J$. We use the notation of the beginning of this subsection.

(1) Suppose $T$ is an $I^{\prime}$-closed subset of $I$ and $T \cap J=H$. Then $T \cap I^{\prime}$ is an $H^{\prime}$-closed subset of $I^{\prime}$.

(2) Suppose $S$ is an $H^{\prime}$-closed subset of $I^{\prime}$ and $T=H \cup S$. Then $T$ is an $I^{\prime}$-closed subset of $I$.

(3) Let $T \subseteq I$ and $S \subseteq I^{\prime}$. Let $T^{\prime}=\mathrm{Cl}_{I^{\prime}, I^{\prime}-S}\left(T \cap I^{\prime}\right), K=\mathrm{K}_{I, I^{\prime}}(T)$, and $K^{\prime}=\mathrm{K}_{I^{\prime}, I^{\prime}-S}\left(T^{\prime}\right)$. Suppose $T \cap S \subseteq K$. Then the graph $D \mid K^{\prime}$ is the union of the connected components of the graph $D \mid\left(K \cap I^{\prime}\right)$ meeting $S$ nontrivially.

(4) Under the hypothesis of Proposition 5.3 the graph $D \mid T_{H}$ is the union of the connected components of $D \mid\left(T \cap I^{\prime}\right)$ meeting $H^{\prime \prime}$ nontrivially.

Lemma 5.5. Suppose hypothesis (A) holds, let $p \in \mathcal{P}$, and let $H \subseteq J$.

(1) Let $\psi_{p, H}: \Gamma(p, H) \rightarrow \Phi(p, H)$ be defined by, for every $U \in \mathcal{O}_{p, H}, \psi_{p, H}(U)=$ $\mathrm{Sh}_{H^{\prime}}\left(U^{\#} \cap p^{\#}\right)$. Then $\psi_{p, H}$ is an isomorphism.

(2) Let $V \in \mathcal{O}, p \in \mathcal{P}_{V}$, and $H \subseteq \operatorname{typ}_{\#}(V) \cap J$. Then $\psi_{p, H}$ induces an isomorphism $\Gamma(p, H)_{V}^{-} \rightarrow \Phi(p, H)_{V_{p, H}}^{-}$.

Proof. Part (1) is immediate from Remark 1.3(5), Lemma 5.4 (1) and (2), and properties (Bld1) and (Bld2) of buildings. To prove (2) we need to show that $U$ is an object of $\Gamma(p, H)_{V}^{-}$if and only if $U^{\prime}:=\operatorname{Sh}_{H^{\prime}}\left(U^{\#} \cap p^{\#}\right)$ is an object of $\Phi(p, H)_{V_{p, H}}^{-}$. Let $S=\operatorname{typ}_{\#}(V)$. Suppose $U$ is an object of $\Gamma(p, H)$, let $N=$ $\operatorname{typ}_{\#}(U)$ and $N^{\prime}=\operatorname{typ}_{\#}\left(U^{\prime}\right)$; we have $N^{\prime}=N-H$. 



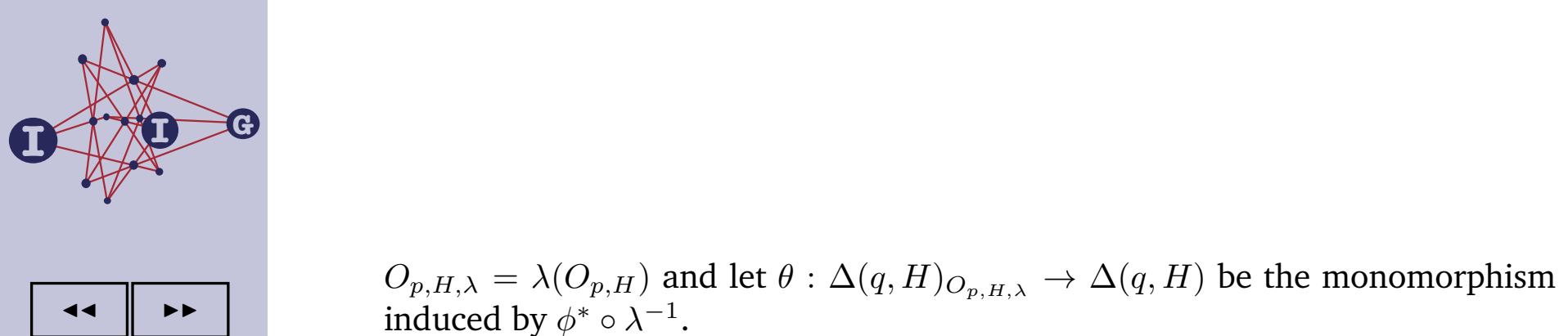
induced by $\phi^{*} \circ \lambda^{-1}$.

Lemma 5.8. Suppose the hypothesis of Proposition 5.3 holds, except possibly for conditions (C1) and (C2), and suppose $|H| \in\{1,2\}$. Then $\theta$ satisfies (Phi-all).

Proof. Let $U$ be an object of $\Phi(q, H)_{O_{p, H, \lambda}}^{-}$and let $S_{0}=$ typ $\#(U)$. Let $S=H \cup S_{0}$, and let $V \in \mathcal{O}$ be such that $\operatorname{typ}^{\#}(V)=S$ and $\left(\lambda^{-1}(U)\right)^{\#} \subseteq V^{\#}$; then by Lemma $5.5 V$ is an object of $\Gamma(p, H)_{O}^{-}$. Let $V^{\prime}=\bar{\phi}(V)$; then $V^{\prime}$ is an object of $\Gamma(q, H)$ and $\operatorname{typ}\left(V^{\prime}\right)=S$. Define $U^{\prime}:=\psi_{q, H}\left(V^{\prime}\right)$. By Lemma 5.5(1) to show that $U^{\prime}$ is as in the conclusion it suffices to show that $\bar{\phi}(\mathcal{A})=\mathcal{A}^{\prime}$, where $\mathcal{A}=\operatorname{Pts}(\Gamma(p, H))_{V}$ and $\mathcal{A}^{\prime}=\operatorname{Pts}(\Gamma(q, H))_{V^{\prime}}$. Since by Lemma $5.1 \phi$ satisfies condition (Phi-all), and the incidence is inclusion, $\bar{\phi}(\mathcal{A}) \subseteq \mathcal{A}^{\prime}$. It remains to prove the reverse inclusion.

Case 1. Suppose $|H|=1$. Then the elements of $\operatorname{Pts}(\Gamma(p, H))$ and $\operatorname{Pts}(\Gamma(q, H))$ are lines of $\Gamma$ with typ \# $_{\text {e }}$ equal to $H$, and all lines of $\Gamma$ incident with $V$ and

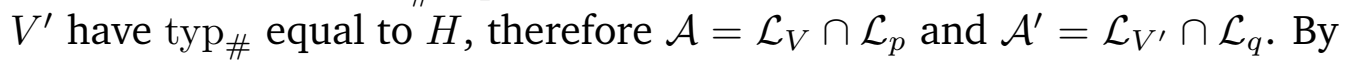
Lemma $5.1 \phi\left(\mathcal{L}_{V}\right)=\mathcal{L}_{V^{\prime}}$, therefore $\phi(\mathcal{A})=\mathcal{A}^{\prime}$.

Case 2. Suppose $|H|=2$. Then the elements of $\operatorname{Pts}(\Gamma(p, H))$ and $\operatorname{Pts}(\Gamma(q, H))$ are planes of $\Gamma$ of the second kind with typ $\mathrm{p}_{\#}$ equal to $H$. Let $\pi^{\prime} \in \mathcal{A}^{\prime}$ and let $\phi_{\pi^{\prime}}$ be the restriction of $\phi^{-1}$ to $\Sigma \mid \mathcal{P}_{\pi^{\prime}}$. Then $\phi_{\pi^{\prime}}$ satisfies the hypothesis of Proposition 3.3. Indeed, let $\Sigma^{\prime}=\left(\mathcal{P}^{\prime}, \mathcal{L}^{\prime}\right)$ be the image of $\Sigma \mid \mathcal{P}_{\pi^{\prime}}$ under $\phi_{\pi^{\prime}}$. Since $\phi$ satisfies condition (Phi-str), $\Sigma^{\prime}$ is a full subgeometry of $\Sigma$ and condition (Phi-lin) holds. By Proposition 3.3 there is a plane $\pi \in \mathcal{A}$, such that $\bar{\phi}(\pi)=\pi^{\prime}$. Therefore $\mathcal{A}^{\prime} \subseteq \bar{\phi}(\mathcal{A})$.

Lemma 5.9. Suppose that the hypothesis of Proposition 5.3 holds and condition (C2) is satisfied, then Part (2) of the conclusion of Proposition 5.3 holds.

Proof. The morphism $\theta$ satisfies the hypothesis of Theorem 3.1. Indeed, by Lemma $5.8 \theta$ satisfies (Phi-str) and, since $\mathcal{B}$ satisfies (Bld-iso) and (Bld-str), so does the building $q$. By Lemma 5.4(4) $\left(H \cup T_{H}\right) \subseteq T$ and, since $H \neq \emptyset$, $\left|T_{H}\right|<T$. Therefore the object $O_{q, H}$ exists by the inductive hypothesis.

\subsection{Intersections of pairs of residues $Q_{q,\{i\}}$}

The object of this section is to prove the following.

Proposition 5.10. Suppose the hypothesis of Proposition 3.4 holds. Let $p \in \mathcal{P}_{O}$ and let $q=\phi(p)$. For every $i \in T \cap J$, let $O_{q,\{i\}}$ be the object of $\Phi(q,\{i\})$ whose existence is asserted in Proposition 5.3. Then $O_{q,\{i\}}^{\#} \cap O_{q,\{j\}}^{\#} \neq \emptyset$ for all $\{i, j\} \subseteq$ $J \cap T$. 



To prove Proposition 3.4(2) we need the following two results. Recall that $\bar{\phi}$ was defined in Corollary 5.2 and $\phi^{*}$ was defined before Lemma 5.7.

Lemma 5.12. Suppose the hypothesis of Proposition 3.4 holds. Let $p \in \mathcal{P}_{O}$, let $q=\phi(p)$, let $O_{q}$ be the object of $\Gamma$ as in (Shd-loc) (existing by Proposition 3.4(1)) and let $R_{q}=q^{\#} \cap\left(O_{q}\right)^{\#}$. Then, for every $H \subseteq T \cap J$ with $|H|=2$, $\phi^{*}$ induces a bijection $\operatorname{Pts}(\Phi(p, H))_{O_{p, H}} \rightarrow \operatorname{Pts}(\Phi(q, H))_{R_{q}}$.

go back

full screen

close

quit

Proof. Suppose $H=\{i, j\}$ and let $\mathcal{P}_{0}=\phi^{*}\left(\operatorname{Pts}(\Phi(p, H))_{O_{p, H}}\right)$.

(1) $\mathcal{P}_{0} \subseteq \operatorname{Pts}(\Phi(q, H))_{R_{q}}$.

Let $\pi \in \operatorname{Pts}(\Gamma(p, H))_{O}$ and let $\pi^{\prime}=\bar{\phi}(\pi)$. Let $L \in \operatorname{Pts}(\Gamma(p,\{i\}))_{O}$ and $N \in$ $\operatorname{Pts}(\Gamma(p,\{j\}))_{O}$ be two distinct lines of $\pi$ on $p$, let $L^{\prime}=\phi(L)$ and $N^{\prime}=\phi(N)$. Then $\left(L^{\prime}\right)^{\#} \cap R_{q} \neq \emptyset,\left(N^{\prime}\right)^{\#} \cap R_{q} \neq \emptyset$, and by Lemma $5.11\left(L^{\prime}\right)^{\#} \cap\left(N^{\prime}\right)^{\#} \neq \emptyset$, therefore by property (Bld2) of buildings $\left(L^{\prime}\right)^{\#} \cap\left(N^{\prime}\right)^{\#} \cap R_{q} \neq \emptyset$. By Lemma $5.1\left(\pi^{\prime}\right)^{\#} \cap q^{\#}=\left(L^{\prime}\right)^{\#} \cap\left(N^{\prime}\right)^{\#} \cap q$, therefore $\left(\pi^{\prime}\right)^{\#} \cap R_{q} \neq \emptyset$.

(2) If $O_{p, H}$ is an object of $\Phi(p, H)$ of the second kind, then $\mathcal{P}_{0}=\operatorname{Pts}(\Phi(q, H))_{R_{q}}$. Let $O_{q, H}$ be the object of $\Phi(q, H)$ existing by Proposition 5.3, such that $\mathcal{P}_{0}=$ $\operatorname{Pts}(\Phi(q, H))_{O_{q, H}}$. Then, comparing typ $\left(R_{q}\right)$ and typ $\#\left(O_{q, H}\right)$, by (1) and [13, Theorem 12.15] or by [4, Proposition 4.5] $\left(O_{q, H}\right)^{\#}=\mathrm{Cl}_{I^{\prime}, H^{\prime}}\left(R_{q}\right)$, therefore (2) holds.

(3) If $O_{p, H}$ is an object of $\Phi(p, H)$ of the first kind, then $\operatorname{Pts}(\Phi(q, H))_{R_{q}} \subseteq \mathcal{P}_{0}$.

If $O_{p, H}$ is an object of $\Phi(p, H)$ of the first kind, then $\left|(D(i) \cup D(j)) \cap\left(T \cap I^{\prime}\right)\right| \leq$ 1. Therefore, up to interchanging $i$ and $j$, there are two possibilities: either $D(i) \cap T \cap I^{\prime}=D(j) \cap T \cap I^{\prime}$ or $\left|D(i) \cap T \cap I^{\prime}\right|=1$ and $D(j) \cap T \cap I^{\prime}=\emptyset$. In both cases

$$
D(i) \cap\left(T \cap I^{\prime}\right)=(D(i) \cup D(j)) \cap\left(T \cap I^{\prime}\right)
$$

Let $\pi^{\prime} \in \operatorname{Pts}(\Gamma(q, H))$ be such that $\left(\pi^{\prime}\right)^{\#} \cap R_{q} \neq \emptyset$. Let $L^{\prime} \in \operatorname{Pts}(\Gamma(q,\{i\}))_{\pi^{\prime}}$. Since $\left(\pi^{\prime}\right)^{\#} \cap q^{\#} \subseteq\left(L^{\prime}\right)^{\#} \cap q^{\#}$ and $\left(\pi^{\prime}\right)^{\#} \cap R_{q} \neq \emptyset$, we have $\left(L^{\prime}\right)^{\#} \cap R_{q} \neq \emptyset$. Therefore $L^{\prime}=\phi(L)$ for some line $L \in \operatorname{Pts}(\Gamma(p,\{i\}))_{O}$. Let $\pi_{L} \in \operatorname{Pts}(\Gamma(p, H))_{O}$ be a plane incident with $L$ and let $\pi_{L}^{\prime}=\bar{\phi}\left(\pi_{L}\right)$. By Lemma $5.1 \operatorname{typ}_{\#}\left(\pi^{\prime}\right)=$ $\operatorname{typ}_{\#}\left(\pi_{L}^{\prime}\right)$. We have $\left(\pi^{\prime}\right)^{\#} \cap q^{\#} \subseteq\left(L^{\prime}\right)^{\#} \cap q^{\#}$ and $\left(\pi_{L}^{\prime}\right)^{\#} \cap q^{\#} \subseteq\left(L^{\prime}\right)^{\#} \cap q^{\#}$. By (1) $\left(\pi_{L}^{\prime}\right)^{\#} \cap R_{q} \neq \emptyset$, therefore by equation (5.1) $\left(\pi^{\prime}\right)^{\#} \cap R_{q}=\left(L^{\prime}\right)^{\#} \cap R_{q}=$ $\left(\pi_{L}^{\prime}\right)^{\#} \cap R_{q}$. This shows $\left(\pi^{\prime}\right)^{\#} \cap\left(\pi_{L}^{\prime}\right)^{\#} \neq \emptyset$, therefore $\pi^{\prime}=\pi_{L}^{\prime}$.

Lemma 5.13. Suppose the hypothesis of Proposition 3.4 holds. Let $\pi$ be a plane of $\Gamma$ incident with two distinct intersecting lines in $\mathcal{L}_{1}$. Then $\pi=\bar{\phi}\left(\pi^{\prime}\right)$ for some plane $\pi^{\prime}$ of $\Gamma_{O}^{-}$.

Proof. Suppose $\{L, N\} \subseteq \mathcal{L}_{1}$ are distinct intersecting lines of $\Gamma$ incident with $\pi$, let $q$ be the common point of $L$ and $N$, let $p=\phi^{-1}(q)$, and let $H=\operatorname{typ}_{\#}(\pi) \cap$ 

(Bld-iso') For all residues $R$ and $Q$ of $\mathcal{B}$, if there is an isomorphisms of diagrams $\xi$ : $M|\operatorname{typ}(R) \rightarrow M| \operatorname{typ}(Q)$, then there is an isomorphism of chamber systems $\xi^{\prime}: R \rightarrow Q$ mapping, for every $i \in \operatorname{typ}(R)$, the edges labelled $\{i\}$ to the edges labelled $\{\xi(i)\}$.

Theorem 6.1. Suppose hypothesis (A) holds, $\mathcal{B}$ has property (Bld-iso'), and $\mathrm{M}=$ $\mathrm{Y}_{l, m, n}$, where $l, m$, and $n$ are integers, $l>0, n>0, m \geq-1$. Let $\phi: \Sigma \mid \mathcal{P}_{O} \rightarrow$ $\Sigma \mid \mathcal{P}_{1}$ be an isomorphism, where $O$ is a residue of $\mathcal{B}$ and $\mathcal{P}_{1}$ is a subspace of $\Sigma$.

(1) Suppose $m \geq 0$ and $J=\left\{m^{\prime}\right\}$ or, else, $m=-1$ and $J=\{-1,1\}$. Then $\mathcal{P}_{1}=\mathcal{P}_{O^{\prime}}$ for a residue $O^{\prime}$ of $\mathcal{B}$ with $M_{O} \cong M_{O^{\prime}}$ and the isomorphism of diagrams takes $\operatorname{typ}_{\#}(O) \cap J$ to typ $\operatorname{ta}_{\#}\left(O^{\prime}\right) \cap J$.

(2) Suppose that $J=\{-l, n\}$ and $\operatorname{typ}(O)=I-\left\{\alpha^{\prime}\right\}$ with $1 \leq \alpha \leq m$. Suppose further that either $M$ is $\mathrm{D}_{k}$ and $k \geq 4$ or, else, $M$ is $\mathrm{E}_{6}$ and $m=1$. Then $\mathcal{P}_{1}=\mathcal{P}_{O^{\prime}}$ for a residue $O^{\prime}$ of $\mathcal{B}$ with $M_{O}=M_{O^{\prime}}$.

Remark 6.2. If in Part (1) of Theorem 6.1 we allow $I_{1}$ or $I_{2}$ to be infinite, then the conclusion fails. Indeed, suppose $I_{1}$ is infinite and let $U$ be an object of $\Gamma$ with typ $\#(U)=I-\{1\}$; then $\mathcal{P}_{U}$ is a maximal singular subspace of $\Sigma$. The geometry $\Sigma \mid \mathcal{P}_{U}$ is a projective space of infinite rank, therefore it has a proper subspace $X$ such that $\Sigma|X \cong \Sigma| \mathcal{P}_{U}$. The subspace $X$ is not the shadow of any object of $\Gamma$.

Under the hypothesis of Theorem 6.1 we let $\Sigma_{1}=\Sigma \mid \mathcal{P}_{1}$.

Lemma 6.3. Suppose the hypothesis of Theorem 6.1 holds, except possibly for the condition (Bld-iso'). Suppose $U$ is an object of $\Gamma$ of the first kind, and $U=O$ or $U$ is in $\Gamma_{O}^{-}$. Then $\phi$ induces an isomorphism $\Sigma\left|\mathcal{P}_{U} \rightarrow \Sigma\right| \mathcal{P}_{U^{\prime}}$, where $U^{\prime}$ is a residue of $\mathcal{B}$ with $M_{U^{\prime}} \cong \mathrm{A}_{k}, k=\left|\operatorname{typ}_{\#}(U)\right|$.

Proof. Let $\Sigma^{\prime}=\left(\mathcal{P}^{\prime}, \mathcal{L}^{\prime}\right)$ be the image of $\Sigma_{U}$ under $\phi$. Since the diagram $M_{U}$ is of type $A_{k}, \Sigma^{\prime}$ is a projective space ([13]). By Remark 1.3(4) $\mathcal{P}_{U}$ is a subspace of $\Sigma \mid \mathcal{P}_{O}$, and by hypothesis $\mathcal{P}_{1}$ is a subspace of $\Sigma$, therefore $\mathcal{P}^{\prime}$ is a subspace of $\Sigma$. The conclusion follows from Remark 1.3(3).

Suppose the hypothesis of Theorem 6.1 holds, except possibly for the condition (Bld-iso'). First, suppose that part (1) of the hypothesis holds and $O$ is an object of $\Gamma$ of the second kind. Then typ $\#(O)=I-\{-1-a, 1+b\}$, where $1 \leq a \leq l$ and $1 \leq b \leq n$, and the maximal singular subspaces of $\Sigma_{O}$ are point shadows of residues of $\mathcal{B}$ of types $A=I-\{-1-a, 1\}$ and $B=I-\{-1,1+b\}$. Here, $a$ and $b$ are integers; for instance if $a=l$ and $b=n-1$, then $\operatorname{typ}^{\#}(O)=I-\{-1-l, 1+n-1\}=I-\{n\}$. Let $A^{\prime}=I-\{-1,1+a\}$, let $B^{\prime}=I-\{-1-b, 1\}$, and let $\mathcal{M}_{-}, \mathcal{M}_{+}, \mathcal{M}_{-}^{\prime}, \mathcal{M}_{+}^{\prime}$ be the sets of objects $U$ of $\Gamma$ 


